\title{
ERRATUM
}

\section{Formation and control of Au and Ag nanoparticles inside borate glasses using femtosecond laser and heat treatment}

Jongho Shin · Kyungsik Jang • Ki-Soo Lim •

Ik-Bu Sohn · Young-Chul Noh · Jongmin Lee

Published online: 18 July 2008

(C) Springer-Verlag 2008

\section{Erratum to: Appl Phys A}

DOI 10.1007/s00339-008-4759-9

Incorrect figures were substituted in place of the intended Figs. 1(a) and 1(b) in this article. The correct Figs. 1(a) and 1(b) appear here.

The online version of the original article can be found under doi:10.1007/s00339-008-4759-9.

J. Shin · K. Jang · K.-S. Lim $(\bowtie)$

BK21 Physics Program and Department of Physics,

Chungbuk National University, Cheongju 361-763, Korea

e-mail: kslim@chungbuk.ac.kr

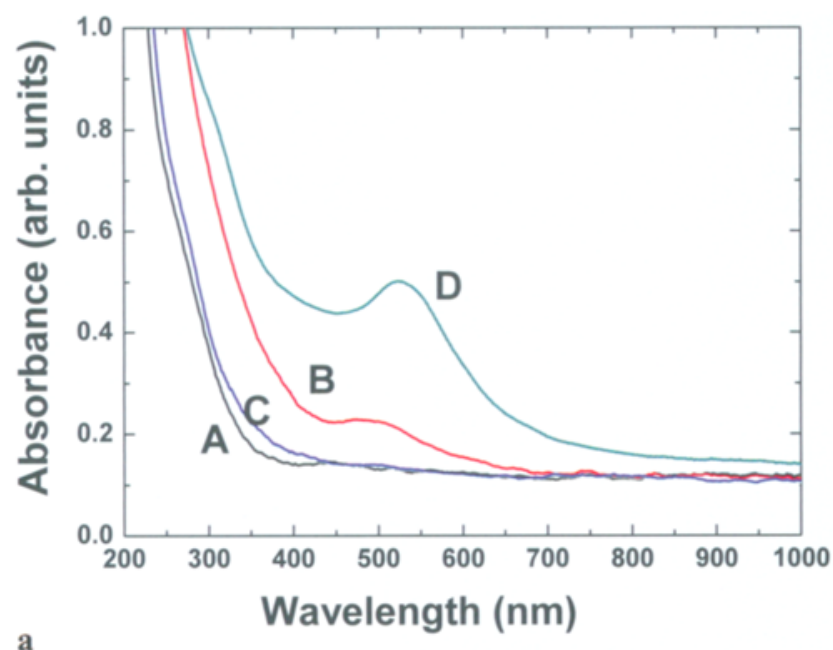

I.-B. Sohn · Y.-C. Noh · J. Lee Advanced Photonics Research Institute, Gwangju Institute of Science and Technology, Gwangju 500-712, Korea

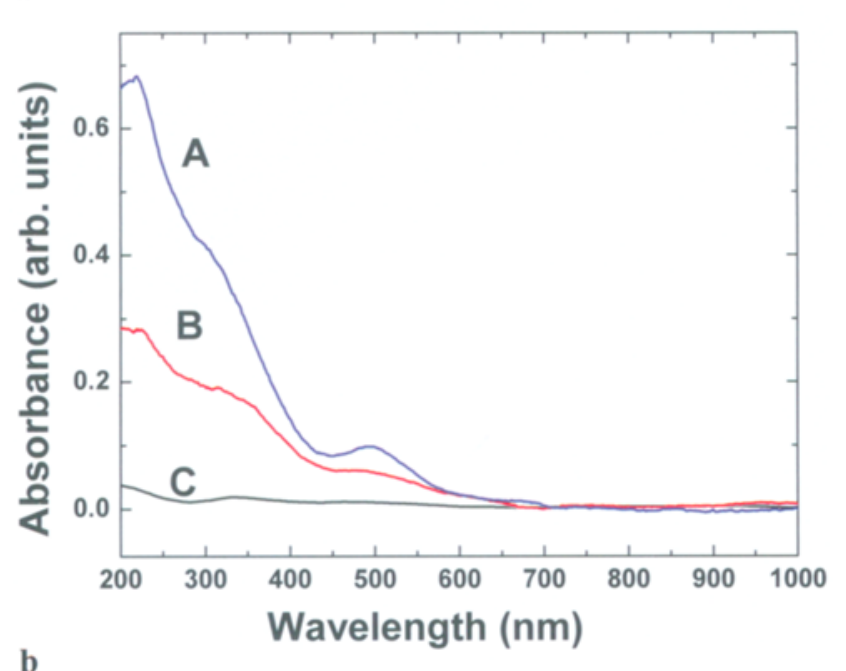

